\title{
Chronic non-bacterial osteitis: imaging review and management implications
}

\begin{abstract}
Chronic Non-Bacterial Osteitis $(\mathrm{CNO})$ is a rare auto inflammatory bone disorder that results in a spectrum of bone lesions. While the pathophysiology of CNO is not well understood, recent studies have shown a derangement on inflammatory markers (such as TNF- $\alpha$, IL-6, IL-20, IL-10 and IL-19). Patients are usually young females and present with localized pain. Since this presentation is nonspecific, a delay in diagnosis and appropriate treatment may occur. Workup shows that patients have normal to moderately elevated inflammatory markers like ESR and CRP. Bone biopsies may be done if the diagnosis is indeterminate and are negative for infectious etiology. Initial imaging usually consists of radiographs at symptomatic sites. Lesions start as osteolysis lesions and progress to sclerotic lesions. Lesions are most commonly found in the metaphysic of the clavicle, pelvis, and the long bones of the lower extremity. If initial imaging studies are negative, then localized MRI is done to look for bone marrow edema. Whole body MRI and bone scan are often used to assess for silent lesions. First line treatment consists of NSAIDs. Second line agents include corticosteroids, TNF- $\alpha$ antagonists, methotrexate, and bisphosphonate. While many patients do well, some patients can unfortunately have persistent symptoms leading to development of arthritis, pathological fractures, limb-length discrepancies, and scoliosis.
\end{abstract}

Volume I Issue 4 - 2017

\author{
Bilal Mujtaba, Rizwan Aslam, Tamara Miner \\ Haygood, John E Madewell, Behrang Amini, \\ Mohamed Elshikh, Gaurav K Synghal \\ Department of Diagnostic Radiology, The University of Texas \\ MD Anderson Cancer Center, USA
}

Correspondence: Bilal Mujtaba, Department of Diagnostic Radiology, The University of Texas MD Anderson Cancer Center, Unit 1475, 1515 Holcombe, Blvd Houston, TX 77030, USA, Tel 7।3-745-9798, Fax 7।3-563-6633, Email bmujtaba@mdandsrson.org

Received: October 20, 2017 | Published: November 30, 2017
Abbreviations: $\mathrm{CNO}$, chronic non-bacterial osteitis; CRMO, chronic recurrent multifocal osteomyelitis; PAPA, pyogenic arthritis pyoderma gangrenosum and acne; DIRA, deficiency of il-1 receptor antagonist; IBD, inflammatory bowel disease; STIR, short-tau inversion recovery; NSAIDS, non steroidal anti-inflammatory drugs

\section{Introduction}

Chronic Non-Bacterial Osteitis (CNO) was first described in 1972 by. ${ }^{1}$ This condition consists of an Autoinflammatory bone disorder that results in bone lesions and is often seen in young children (approximately 7-12 years old). ${ }^{2}$ Patients may suffer from a single to multiple bone lesions that may self-resolve, persist, and/or reoccur. ${ }^{3}$ When patients have the most severe manifestation of $\mathrm{CNO}$ with multiple, reoccurring lesions, this condition is also known as chronic recurrent multifocal osteomyelitis (CRMO). ${ }^{2} \mathrm{CNO}$ is classified as an orphan disease (ORPHA: 324964, OMIM: 259680) with a study in Germany showing an incidence of $0.45 / 100,000$ children. ${ }^{4}$ Patients typically have a nonspecific initial presentation. Often, they present with localized pain $+/$ - swelling. ${ }^{2-5}$ Since this presentation is common in other conditions like bacterial osteomyelitis, bone malignancies, fibrous dysplasia, and/or Langerhans cell histiocytosis, a delay in diagnosis and appropriate treatment may occur. One study showed a delay of diagnosis for $17.3 \pm 24.8$ months. ${ }^{5}$ Therefore, initiating workup for $\mathrm{CNO}$ requires an astute clinical suspicion by general practitioners. Timely suspicion can lead to appropriate workup that can improve the management, reduce unnecessary biopsies, reduce unnecessary imaging studies and reduce unnecessary antibiotic use.

\section{Pathophysiology}

As opposed to autoimmune disorders which may see a derangement in T cells levels and autoantibody levels, $\mathrm{CNO}$ is an auto inflammatory disorder with dysregulation of the innate immune system. While the exact pathophysiology of $\mathrm{CNO}$ is not fully understood, recent studies have suggested derangement in various cytokine levels as potential causes for the bone lesions. ${ }^{3-6}$ In individuals with CRMO, there was evidence of increased pro-inflammatory markers such as TNF- $\alpha$, IL6 , and IL-20 and decreased expression of anti-inflammatory markers such as IL-10 and IL-19. ${ }^{6,7}$ Additionally, animal studies showed a link between IL-10 deficiency, increased NLRP3 Inflammasome activity, and bone erosions. ${ }^{8}$ Pro-inflammatory cytokines can also upregulate RANKL which leads to increased levels of osteoclasts. ${ }^{9}$ Thus, this suggests a possible connection to the cytokine imbalances leading to the bone lesions. ${ }^{6}$ Furthermore, there is some thought that certain bacteria like Propionibacterium acnes may act as a trigger for an inflammatory cascade that can lead to $\mathrm{CNO}$ in susceptible individuals. ${ }^{10-15} \mathrm{P}$. acnes may cause increased activation of the Inflammasome and proinflammatory cytokines (such as IL-1 $\beta$ and IL-18) that can potentially lead to the bone lesions, but this is still relatively controversial and more studies need to be done. ${ }^{16-18}$ Additionally, there seems to be some susceptibility based of genetics as well. Majeed syndrome is a disorder where patients develop anemia, recurrent fevers, and early onset $\mathrm{CNO}^{3-10}$ This syndrome has been localized to mutations in LPIN2 gene. ${ }^{11}$ Additionally, other genetic autoinflammatory bone syndromes have also been identified such as DIRA (deficiency of IL-1 receptor antagonist), PAPA (pyogenic arthritis, pyoderma gangrenosum and acne), and cherubism. ${ }^{3-10}$

\section{Clinical features/workup}

Patients usually have nonspecific clinical findings. They are usually young, female (2:1 female to male ratio) and present with localized bone pain usually in the lower extremities, clavicle, and/or pelvis ${ }^{12}$. Only about $33 \%$ of patients have low grade fevers. ${ }^{13}$ There is an association with other autoimmune and auto inflammatory disorders like inflammatory bowel disease (IBD), psoriasis, and palmar plantar pustulosis. ${ }^{14}$ Additionally, SAPHO (synovitis, acne, pustulosis, hyperostosis, osteitis) syndrome is thought to be a similar, possibly related, condition in which patients have bone lesions 
along with characteristic skin findings of palm plantar pustulosis (blistering of the palms and soles). SAPHO patients present as adults rather than children. Workup usually consists of imaging of the symptomatic site and laboratory studies like CBC, ESR, and CRP. Values of the inflammatory markers range from normal to moderately elevated. ${ }^{15,16}$ When inflammatory markers and WBC count were severely elevated, there is an increase concern for malignancy or infection..$^{16}$ The decision whether to perform a bone biopsy is more varied among practitioners. In a recent study assessing the diagnostic approach pediatric rheumatologist utilize in diagnosing $\mathrm{CNO}$, the clinicians tended to perform bone biopsies when patients presented with solitary bone lesions, constitutional symptoms (fever, weight loss, night sweats), and nocturnal bone pain as they had increased suspicion for malignancy or infection. ${ }^{17}$ However, when patients had multifocal lesions in classic sites or had associated conditions like IBD or psoriasis, the clinicians tended to avoid bone biopsy [17]. Additionally created a scoring system to determine whether to obtain a bone biopsy. In this scoring system, Jansson et al. designated a score coefficient to several risk factors such as normal blood cell count: 13 points, symmetric lesions: 10 points, lesions with marginal sclerosis: 10 points, normal body temperature: 9 points, vertebral, clavicular, or sternal lesions: 8 points, radiologically proven lesions $\geq 2: 7$ points, and CRP $\geq 1: 6$ points. ${ }^{17}$ Patients with a score $\geq 39$ had a positive predictive value of $97 \%$ for $\mathrm{CNO}$, and a score $\leq 28$ had a negative predictive value of $97 \% .{ }^{17}$ Recommended bone biopsy only in the individuals with a single lesion and a score $\leq 28$. Score values between 29-38 were indeterminate and require close clinical monitoring ${ }^{18}$ also created the Bristol diagnostic criteria based off a cohort study from children diagnosed with CRMO at Bristol Royal Hospital for Children to reduce delay in diagnosis. ${ }^{19}$ Based off this criteria, CNO can be diagnosed with the 1) presence of typical clinical findings, 2) the presence of typical radiological findings and either 3) $>1$ bone lesion with $\mathrm{CRP}<30 \mathrm{~g} / \mathrm{L}$ or 4 ) unifocal disease and/or $\mathrm{CRP}>30$ with negative bacterial growth on bone biopsy, ${ }^{19}$ (Figures 1-4).
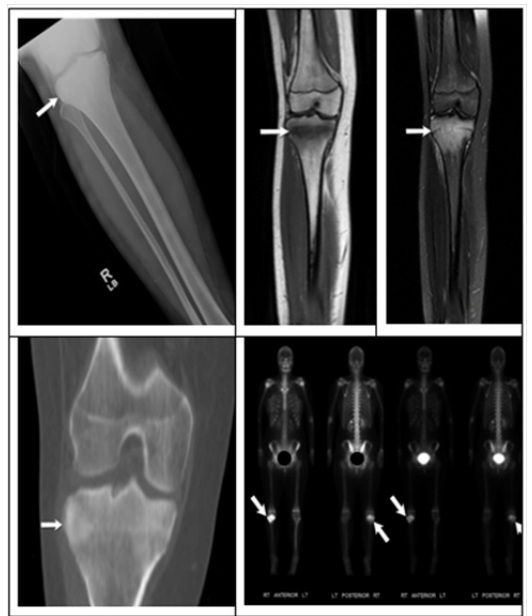

Figure I Patient I Imaging review.

a. Figure I(A) AP view X-ray of the tibia demonstrating increased sclerosis of the proximal tibia.

b. Figure I(B) Coronal TI MRI showing bone marrow edema and cortical sclerosis.

c. Figure I(C) Coronal T2 MRI showing bone marrow edema and cortical sclerosis.

d. Figure I(D) Coronal non-contrast CT scan demonstrating increased sclerosis in the proximal tibia.

e. Figure I(E) Tc $99 \mathrm{~m}$ Bone scan showing increased uptake in the right proximal tibia.
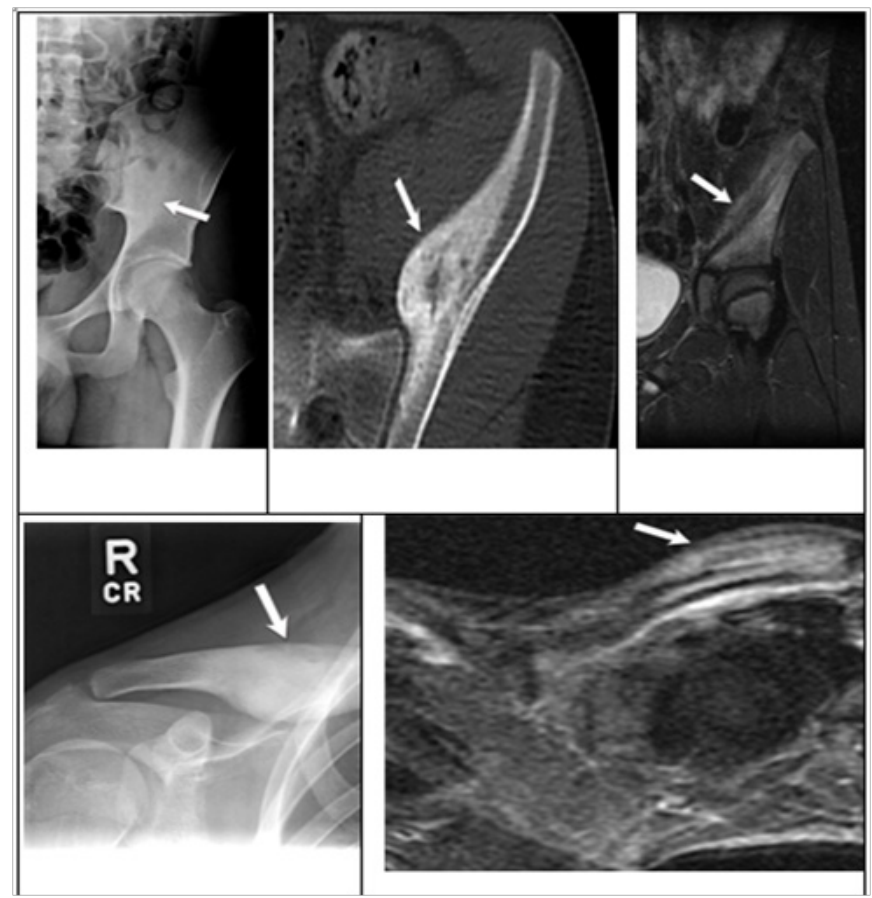

Figure 2 Patient 2 imaging review.

a. Figure 2A:AP view $X$-ray of pelvis showing a sclerotic left iliac lesion.

b. Figure 2B:Axial CT demonstrating sclerotic left iliac lesion.

c. Figure 2C:T2 MRI of the pelvis demonstrating left iliac cortical sclerosis and underlying bone marrow edema.

d. Figure 2D:AP view $X$-ray of the right clavicle showing increased sclerosis. Findings consistent with multifocal $\mathrm{CNO}$ in the same patient.

e. Figure 2E: $\mathrm{MRI}$ of the clavicle showing cortical sclerosis and bone marrow edema.

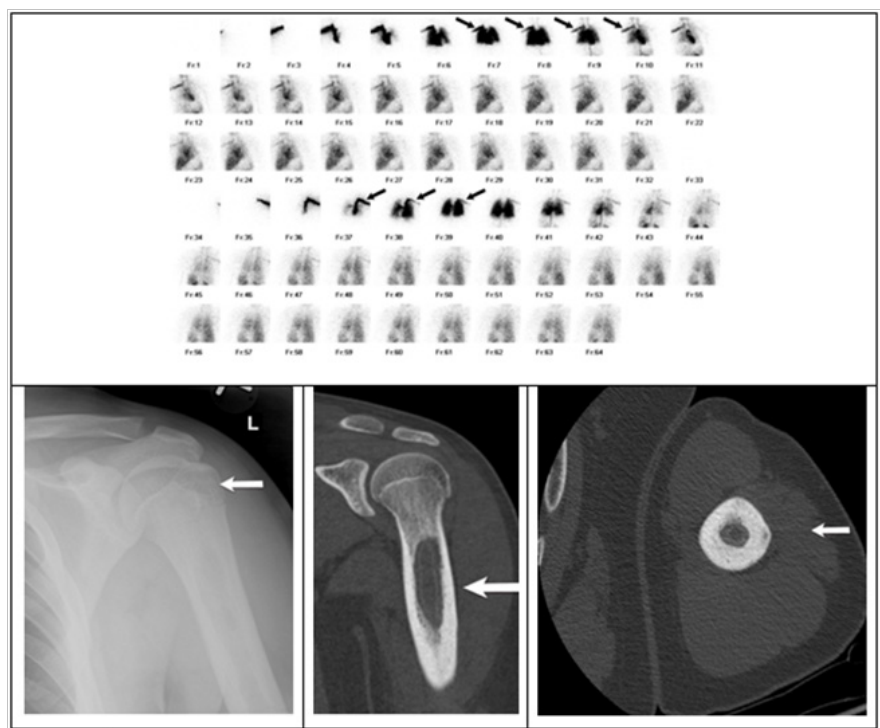

Figure 3 Patient 3 imaging review.

a. Figure $3 A$ :AP view $X$-ray of the left hummers showing cortical sclerosis.

b. Figure 3B: Coronal and axial CT scans showing cortical sclerosis with intramedullary calcifications consistent with long staging sclerotic reparative lesion with both endosteal and periosteal components.

c. Figure 3C: 3 phase bone scan demonstrating increased radiotracer activity in the proximal to mid-hummers. 


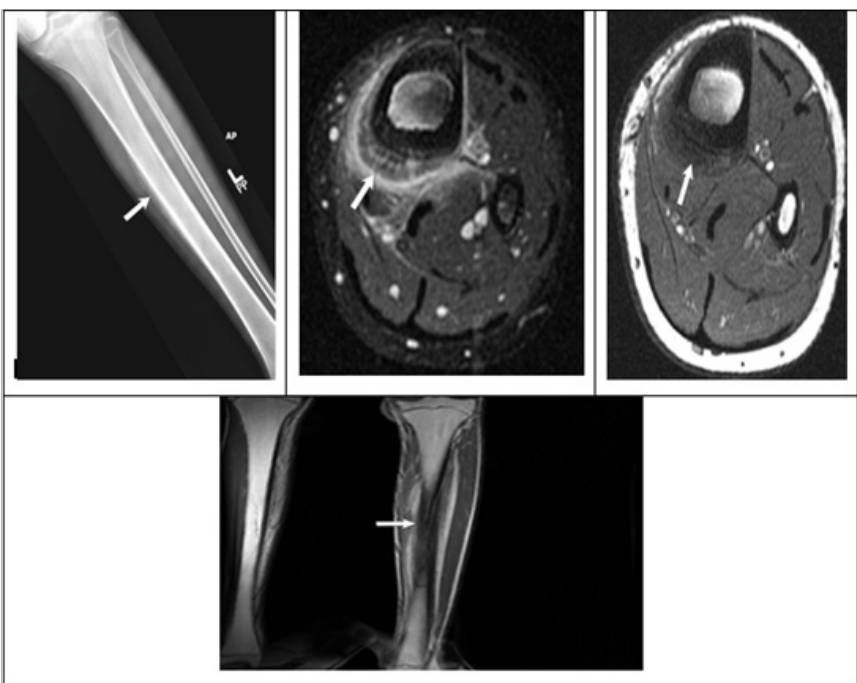

Figure 4 Patient 4 imaging review.

a. Figure 4A: One view $X$-ray showing chronic thickening and mature periosteal reaction.

b. Figure 4B:Axial TI post contrast demonstrating bone marrow edema and ongoing maturing periosteitis.

c. Figure 4C:Axial TI shows cortical thickening and bone marrow edema.

d. Figure 4D: Coronal TI demonstrating sclerosis and mature periosteal reaction.

\section{Image findings}

The most common imaging studies ordered is conventional radiographs $(89 \%)$, local MRIs (79\%), and bone scintigraphy (43\%) and whole body MRI (36\%). ${ }^{17}$ Therefore, initial imaging consists of radiographs at symptomatic sites. Bone lesions start as osteolytic lesions. Over time, the images develop sclerosis around the osteolytic lesions. Eventually, the lesions may become predominately sclerotic. ${ }^{20}$ Bone lesions can occur anywhere throughout the body. However, they tend to have a predisposition to the metaphyseal region of bones $(75 \%){ }^{20}$ Additionally, common sites of involvement include the clavicle $(34 \%)$, pelvis $(34 \%)$, tibia $(31 \%)$, and femur $(31 \%) .{ }^{15}$ While there are no pathognomonic imaging findings for $\mathrm{CNO}$, clavicle lesions tend to be strongly associated with $\mathrm{CNO}$ as clavicle is usually spared during bacterial osteomyelitis. ${ }^{20}$

Another characteristic site for $\mathrm{CNO}$ is the mandible, which is seen in approximately $5 \%$ of patients. ${ }^{20}$ Lytic lesions can cause disruption of the cortical and trabecular bone and subsequent sclerosis can eventually cause enlargement of the mandible. If the initial studies are negative, then localized MRI is done to look for bone marrow edema (decreased signal on T1 weighted images and increased signal on T2 weighted images. ${ }^{20}$ While patients may initially present with a single site of painful bone involvement, studies have shown that patient have, on average, $\sim 3.5$ bony lesions. ${ }^{15-21}$ To evaluate these silent lesions, bone scintigraphy and/or whole-body MRI are used. Bone scintigraphy uses Technetium $99 \mathrm{~m}$ to identify areas of bone remodeling. Thus, bone scintigraphy has been shown to be sensitive to determine clinically silent lesions. ${ }^{22}$

Additionally, it can better identify spinal and pelvic lesions that are difficult to assess via X-Rays. ${ }^{22}$ Lesions will show initial hyperemia with focal areas of increased uptake on delayed phase images. ${ }^{22}$ Bone scintigraphy tends to be more readily available and less expensive than whole-body MRIs. ${ }^{20}$ However, whole body MRI has been shown to have improved sensitivity. ${ }^{23}$ Therefore, MRI is now being utilized at an increasing rate to evaluate for silent lesions. On MRI, the lesions may be visible along with marrow edema and surrounding soft-tissue inflammations. Whole body MRI use short-tau inversion recovery (STIR) and T1-weighted pulse sequences for evaluation. ${ }^{23,24}$ Abnormal lesions have increased signal on the STIR sequence and decreased signal on the T1 weighted sequence. ${ }^{23,24}$ Patients are often having follow up imaging to assess for the status of known lesions and to assess for any new lesions. ${ }^{25}$ Whole body MRI is recommended, but bone scintigraphy often is done due to increased availability.

\section{Histopathology}

Bone biopsy shows evidence of inflammation without the presence of microorganisms. Initially, there are increased polymorphonuclear leukocytes, but later stages have increased lymphocytes. ${ }^{26}$ Occasionally, bone cultures may be positive for P. acnes. However, this is usually considered to be a contaminant rather than a direct bacterial cause of the patient lesions. ${ }^{3-27}$

\section{Treatment options}

Many patients are started on antibiotics due to misdiagnosis of bacterial osteomyelitis. This can cause unnecessary hospitalization (IV antibiotics) or prolonged oral antibiotic regimens. One study showed that $54 \%$ of the patients had received at least one course of IV antibiotics before diagnosis. ${ }^{19}$ Not only does this cause an unnecessary medical burden, it delays the appropriate diagnosis and appropriate treatment.

Unfortunately, an evidence based treatment protocol has not been well defined as current treatment regimens are empiric based. Generally, the first line treatment is non steroidal anti-inflammatory drugs (NSAIDS). ${ }^{3}$ Some studies have shown up to $80 \%$ success with NSAIDs. ${ }^{28}$ However, some studies may contest the effectiveness of NSAIDs as they may not effectively treat the underlying problem. One study showed while there was improved symptom relief and overall reduction in lesions after starting naproxen therapy, $41 \%$ of the patients still developed new lesions detected on whole body MRI. More research and randomized control trials need to be done to understand the true effectiveness of NSAIDs in treating and preventing new lesions. ${ }^{25}$

There is no consensus on second-line agents, but some options include corticosteroids, TNF- $\alpha$ antagonist, DMARDs like methotrexate, and bisphosphonates ${ }^{3}$. Bisphosphonate therapy like pamidronate and alendronate is encouraging and has shown patient improvement especially with mandibular lesions. ${ }^{29-36}$ However, the long-term side effects in children have not been well studied, which has somewhat limited increased adoption. Ultimately, treatment is done to improve prognosis and prevent the complications of the bone lesions and provide symptomatic pain relief.

\section{Prognosis}

Most patients usually have a good long term prognosis. ${ }^{31}$ A study by showed a $78 \%$ of patients after 13 years of follow up had a score of 0 measures by the Health Assessment Questionnaire (HAQ) suggesting no physical disability. ${ }^{31}$ Another study by showed a HAQ of 0 in $59 \%$ of patients. ${ }^{21}$ However, the study by also demonstrated 
that patients had CNO lesion for median of 5.7 years and about $25 \%$ of patients had persistent symptoms more than 12 years. Much of the sequelae of CNO depend on the location of the lesions. Many patients end up developing arthritis. ${ }^{25}$ Lesions near the growth plate can lead to their premature closure resulting in limb-length discrepancy, which should be treated immediately to minimize morbidity. ${ }^{32}$ Pathological fractures can occur when the integrity of the bones is compromised. ${ }^{33}$ Lesions in the spine can lead to kyphosis, scoliosis, and up to $40 \%$ may develop vertebral fractures. ${ }^{33}$ Mandibular lesions can result in paresthesia and their extension to the Temporomandibular joint can lead to trismus. ${ }^{37}$

\section{Conclusion}

While CNO diagnosis often requires a multidisciplinary approach, general practitioners and pediatricians will often be the first point of contact for many of the patients that suffer from bone pain. Thus, early recognition of CNO by these individuals can lead to prompt treatment with NSAIDs and avoid costly hospitalizations, antibiotics, unnecessary bone biopsies, and improper imaging studies. It can also help prevent more serious complications like limb-length discrepancies and pathological fractures.

\section{Acknowledgements}

None.

\section{Conflict of interest}

The author declares no conflict of interest.

\section{References}

1. Giedion A, Holthusen W, Masel LF, et al. Subacute and chronic symmetrical osteomyelitis. Ann Radiol (Paris). 1972;15(3):329-342.

2. Hedrich CM, Hahn G, Girschick HJ, et al. A clinical and pathomechanistic profile of chronic nonbacterial osteomyelitis/chronic recurrent multifocal osteomyelitis and challenges facing the field. Expert Rev Clin Immunol. 2013;9(9):845-854.

3. Stern SM, Ferguson PJ. Autoinflammatory bone diseases. Rheum Dis Clin North Am. 2013;39(4):735-749.

4. Grote V, Silier CC, Voit AM, et al. Bacterial osteomyelitis or nonbacteria osteitis in children: A study involving the german surveillance unit for rare diseases in childhood. Pediatr Infect Dis J. 2017;36(5):451-456.

5. Wipff J, Costantino F, Lemelle I, et al. A large national cohort of French patients with chronic recurrent multifocal osteitis. Arthritis Rheumatol. 2015;67(4):1128-1137.

6. Hofmann SR, Kapplusch F, Mabert K, et al. The molecular pathophysiology of chronic non-bacterial osteomyelitis (CNO)-a systematic review. Mol Cell Pediatr. 2017;4(1):7 p.

7. Hofmann SR, Morbach H, Schwarz T, et al. Attenuated TLR4/MAPK signaling in monocytes from patients with CRMO results in impaired IL-10 expression. Clin Immunol. 2012;145(1):69-76.

8. Greenhill CJ, Jones GW, Nowell MA, et al. Interleukin-10 regulates the inflammasome-driven augmentation of inflammatory arthritis and joint destruction. Arthritis Res Ther. 2014;16(4):419.

9. Nakashima T, Takayanagi H. Osteoclasts and the immune system. J Bone Miner Metab. 2009;27(5):519-529.

10. Morbach H, Hedrich CM, Beer M, et al. Autoinflammatory bone disorders. Clin Immunol. 2013;147(3):185-196.
11. Al Mosawi ZS, Al Saad KK, Ijadi Maghsoodi R, et al. A splice site mutation confirms the role of LPIN2 in majeed syndrome. Arthritis Rheum. 2007;56(3):960-964.

12. Borzutzky A, Stern S, Reiff A, et al. Pediatric chronic nonbacterial osteomyelitis. Pediatrics. 2012;130(5):1190e-1197e.

13. Schultz C, Holterhus PM, Seidel A. Chronic recurrent multifocal osteomyelitis in children. Pediatr Infect Dis J. 1999;18(11):1008-1013.

14. Ferguson PJ, Sandu M. Current understanding of the pathogenesis and management of chronic recurrent multifocal osteomyelitis. Curr Rheumatol Rep. 2012;14(2):130-141.

15. Wintrich S, Horneff G. Characteristics and outcomes of chronic nonbacterial osteitis in children. Eur J Rheumatol. 2015;2(4):139-142.

16. Hedrich CM, Hofmann SR, Pablik J, et al. Autoinflammatory bone disorders with special focus on chronic recurrent multifocal osteomyelitis (CRMO). Pediatr Rheumatol Online J. 2013;11(1):11-47.

17. Zhao Y, Dedeoglu F, Ferguson PJ, et al. Physicians' perspectives on the diagnosis and treatment of chronic nonbacterial osteomyelitis. Int $J$ Rheumatol. 2017;2017:7694942 p.

18. Jansson AF, Muller TH, Gliera L, et al. Clinical scores for nonbacterial osteitis in children and adults. Arthritis Rheum. 2009;60(4):1152-1159.

19. Roderick MR, Shah R, Rogers V, et al. Chronic recurrent multifocal osteomyelitis (CRMO)-advancing the diagnosis. Pediatr Rheumatol Online J. 2016;14(1):47.

20. Khanna G, Sato TSP, Ferguson P. Imaging of chronic recurrent multifocal osteomyelitis. Radiographics. 2009;29(4):1159-1177.

21. Catalano Pons C, Comte A, Wipff J, et al. Clinical outcome in children with chronic recurrent multifocal osteomyelitis. Rheumatology (Oxford). 2008;47(9):1397-1399.

22. Acikgoz G, Averill LW. Chronic recurrent multifocal osteomyelitis: Typical patterns of bone involvement in whole-body bone scintigraphy. Nucl Med Commun. 2014;35(8):797-807.

23. Fritz J. The contributions of whole-body magnetic resonance imaging for the diagnosis and management of chronic recurrent multifocal osteomyelitis. J Rheumatol. 2015;42(8):1359-1360.

24. Fritz J, Tzaribatchev N, Claussen CD, et al. Chronic recurren multifocal osteomyelitis: Comparison of whole-body MR imaging with radiography and correlation with clinical and laboratory data. Radiology. 2009;252(3):842-851.

25. Beck C, Morbach H, Beer M, et al. Chronic nonbacterial osteomyelitis in childhood: Prospective follow-up during the first year of antiinflammatory treatment. Arthritis Res Ther. 2010;12(2):74R.

26. Bj0rksten B, Boquist L. Histopathological aspects of chronic recurrent multifocal osteomyelitis. J Bone Joint Surg Br. 1980;62(3):376-380.

27. Winters R, Tatum SA. Chronic nonbacterial osteomyelitis. Curr Opin Otolaryngol Head Neck Surg. 2014;22(4):332-335.

28. Twilt M, Laxer RM. Clinical care of children with sterile bone inflammation. Curr Opin Rheumatol. 2011;23(5):424-431.

29. Roderick M, Shah R, Finn A, et al. Efficacy of pamidronate therapy in children with chronic non-bacterial osteitis: Disease activity assessment by whole body magnetic resonance imaging. Rheumatology (Oxford). 2014;53(11):1973-1976.

30. Hirano D, Chiba K, Yamada S, et al. Oral alendronate in pediatric chronic recurrent multifocal osteomyelitis. Pediatr Int. 2017;59(4):506-508.

31. Huber AM, Lam PY, Duffy CM, et al. Chronic recurrent multifocal osteomyelitis: Clinical outcomes after more than five years of follow-up. J Pediatr. 2002;141(2):198-203. 
32. Duffy CM, Lam PY, Ditchfield M, et al. Chronic recurrent multifocal osteomyelitis: Review of orthopaedic complications at maturity. $J$ Pediatr Orthop. 2002;22(4):501-505.

33. Jansson AF, Grote V, ESPED Study Group. Nonbacterial osteitis in children: Data of a german incidence surveillance study. Acta Paediatr. 2011;100(8):1150-1157.

34. Sharma M, Ferguson PJ. Autoinflammatory bone disorders: Update on immunologic abnormalities and clues about possible triggers. Curr Opin Rheumatol. 2013;25(5):658-664.
35. Patel R, Jacob R, Lee $\mathrm{K}$, et al. Parotid swelling and chronic recurrent multifocal osteomyelitis of mandible in children. Int $J$ Pediatr Otorhinolaryngol. 2015;79(1):47-52.

36. Compeyrot Lacassagne S, Rosenberg AM, Babyn P, et al. Pamidronate treatment of chronic noninfectious inflammatory lesions of the mandible in children. $J$ Rheumatol. 2007;34(7):1585-1589.

37. Soubrier M, Dubost JJ, Ristori JM, et al. Pamidronate in the treatment of diffuse sclerosing osteomyelitis of the mandible. Oral Surg Oral Med Oral Pathol Oral Radiol Endod. 2001;92(6):637-640. 